\title{
Classifying general nonlinear force laws in cell-based models via the continuum limit
}

\author{
Philip J. Murray, ${ }^{1}$ Carina M. Edwards, ${ }^{2}$ Marcus J. Tindall, ${ }^{3}$ and Philip K. Maini ${ }^{1,4}$ \\ ${ }^{1}$ Centre for Mathematical Biology, Mathematical Institute, 24-29 St Giles', Oxford OX1 3LB, United Kingdom \\ ${ }^{2}$ Center for Modeling and Simulation in the Biosciences, Im Neuenheimer Feld 267, University of Heidelberg, \\ D-69120 Heidelberg, Germany \\ ${ }^{3}$ School of Biological Sciences, Department of Mathematics and Statistics, and Institute for Cardiovascular and Metabolic Research, \\ University of Reading, Whiteknights, Reading, Berkshire RG6 6AJ, United Kingdom \\ ${ }^{4}$ Oxford Centre for Integrative Systems Biology, Department of Biochemistry, South Parks Road, Oxford OXI 3QU, United Kingdom
}

(Received 6 September 2011; published 23 February 2012)

\begin{abstract}
Although discrete cell-based frameworks are now commonly used to simulate a whole range of biological phenomena, it is typically not obvious how the numerous different types of model are related to one another, nor which one is most appropriate in a given context. Here we demonstrate how individual cell movement on the discrete scale modeled using nonlinear force laws can be described by nonlinear diffusion coefficients on the continuum scale. A general relationship between nonlinear force laws and their respective diffusion coefficients is derived in one spatial dimension and, subsequently, a range of particular examples is considered. For each case excellent agreement is observed between numerical solutions of the discrete and corresponding continuum models. Three case studies are considered in which we demonstrate how the derived nonlinear diffusion coefficients can be used to (a) relate different discrete models of cell behavior; (b) derive discrete, intercell force laws from previously posed diffusion coefficients, and (c) describe aggregative behavior in discrete simulations.
\end{abstract}

DOI: 10.1103/PhysRevE.85.021921

PACS number(s): 87.17.Rt, 87.18.Fx

\section{INTRODUCTION}

The modeling of multicellular systems has applications across a range of life science disciplines, from biofilm formation to tumor growth and, naturally, a whole host of different mathematical modeling techniques have been employed in different contexts. However, as the use of mathematical models in the life sciences becomes more common, so the need to clearly distinguish between and relate particular modeling frameworks becomes increasingly important. In many cases, the only tool available to perform such comparisons is brute force numerical computation but as parameters are often not comparable across different models, it can be difficult to gain qualitative insight and make generalizations based solely upon simulation results.

One approach to modeling cell populations, which provides a natural platform in which cell-level properties, such as elasticity, adhesion, motility, and cell proliferation, can be related to experimental measurements, is to treat the cells, or parts thereof, as discrete entities. The discrete models can easily account for important biological phenomena, such as heterogeneity between cells within a population or the effect of noise at various scales. Discrete cell-level models can themselves, broadly speaking, be separated into two categories: on- and off-lattice. In traditional cellular automata (CA) each biological cell is represented by a single grid point and automaton rules are chosen that simulate a particular biological phenomenon [1]. The use of CA is widespread in biological modeling (e.g., [2-7]) with the main advantages being ease of implementation and computational efficiency at large numbers of cells. However, it can be difficult to relate automaton rules to biomechanics, primarily as a result of the restriction of cell locations to discrete lattice points. Moreover, the lattice can induce artifacts into simulation results.
Another class of on-lattice discrete models is the cellular Potts model, in which a cell is represented by a number of lattice points. Using the Metropolis algorithm, a given cell population is assumed to minimize a global energy function which is defined such that individual cells exhibit particular phenomena, such as volume conservation or chemotactic movement [8]. The higher resolution of cells in the cellular Potts model compared to traditional cellular automata allows certain biologically relevant quantities, such as a cell's boundary area, to be simulated and, relative to CA, lattice artifacts are greatly reduced. However, in comparison with CA, the additional resolution of a given cell on the $\mathrm{CA}$ lattice increases the computational load associated with the simulations.

In contrast to cellular automata, cells in off-lattice models can occupy positions in continuous space (e.g., [9-13]). Cell positions are updated by balancing physically motivated forces and solving Newton's second law in the overdamped limit. Neglecting cell-cell friction, the equation of motion for the $i$ th cell in a population is given by an equation of the form

$$
\eta \dot{\mathbf{r}_{i}}=\sum_{j} \mathbf{F}_{i j}, \quad i=1, \ldots, N,
$$

where $\mathbf{r}_{i}$ represents the cell position, $\eta$ is the cell damping constant, $\mathbf{F}_{i j}$ is the force exerted on the $i$ th cell by the $j$ th cell, the sum is taken over nearest neighbors, and $N$ is the number of cells in the system $[12,14,15]$. The damping term is assumed to originate from cell-matrix or internal cell friction. Among the advantages of the off-lattice models is that experimentally testable aspects of cell-cell mechanics can be incorporated in the force laws.

The force law in Eq. (1) is chosen to capture specific interactions between pairs of cells and typically accounts for elastic repulsion, as cells approach one another, and attractive adhesion, owing to surface adhesion molecules. One of the 
simplest representations of the elastic and adhesive forces is to model them using a linear force law $[12,16]$. However, a limitation of this approach is that the rate at which the repulsive force between a pair of neighboring cells increases as they approach one another is not sufficiently large. Hence, variations have been considered in which the force between a pair of cells is linear at large separations but exponentially increases for low separations [17]. Even with this modification, the linear force regime can be criticized as there is not a natural interpretation of the spring constant for a complex object such as a cell. Hence, the Hertz model of elastic contact between nonadhering spheres and variations thereof are often used to model elastic cell interactions $[11,14]$. Here the model parameters are physically measurable quantities, such as the modulus of elasticity and Poisson's ratio. As well as mechanically motivated models of cell-cell interactions, phenomenological models that exhibit the properties of large short-range repulsion and weak long-range attraction, such as the Lennard-Jones force law, have been used to simulate cellcell interactions (e.g., [18,19]) in the off-lattice framework. However, such models have their origins in the approximation of molecular interactions and, as such, it is not obvious how they can be parametrized in biological systems.

A problem with discrete descriptions of cell behavior is that they can become computationally inefficient at the large numbers of cells required to represent many biological systems of interest. Moreover, metrics for relating population-scale measurements to cellular-scale model details are limited. By deriving continuum models these problems can, to a certain extent, be overcome: the resulting partial differential equations (PDEs) can be solved to simulate large numbers of cells and traditional applied mathematics techniques, such as perturbation and bifurcation theory, can be used to analyze the continuum models, at least in relevant limits. Thus, in particular limiting cases, continuum approximations can be used to develop insight into the behavior of the underlying simulations and the biological problem.

The coarse-graining of discrete models to derive continuum equations that yield further insight into discrete model behavior is becoming an increasingly utilized technique in biological modeling [16,20-27]. The details of the particular coarse-graining technique used depend strongly on the type of discrete model under consideration, but an emerging theme is that nonlinear diffusion equations can provide a means of analyzing and categorizing discrete simulations: for example, Alber and co-workers [21,22] have derived limiting nonlinear PDEs which describe cell chemotaxis and adhesion in the cellular Potts model; Simpson and co-workers [26,27] have derived nonlinear diffusion equations that describe stochastic cellular automaton models at the population scale; Bodnar and Velazquez [25] have shown that a porous medium equation can describe the behavior of a system of particles interacting via a repulsive potential; and Murray et al. [16], considering the special case of an off-lattice linear spring-based model in one spatial dimension, have shown that the discrete equations of motion transform into a nonlinear diffusion equation.

In this paper we generalize the linear-spring model derived by Murray et al. [16] to the case of a general nonlinear force law. Our key result is that if cells interact via an equation of motion of the form given by Eq. (1) then the cell density at the continuum scale obeys the nonlinear diffusion equation

$$
\frac{\partial q}{\partial \tau}=\frac{\partial}{\partial r}\left(D(q) \frac{\partial q}{\partial r}\right)
$$

where $q$ is the cell number density, with the corresponding nonlinear diffusion coefficient given by

$$
D(q)=-\left.\frac{\left|r_{i}-r_{j}\right|^{2}}{\eta} F^{\prime}\left(\left|r_{i}-r_{j}\right|\right)\right|_{\left|r_{i}-r_{j}\right|=1 / q}=-\frac{1}{\eta q^{2}} F^{\prime}\left(\frac{1}{q}\right),
$$

where the prime denotes differentiation with respect to $\left|r_{i}-r_{j}\right|$.

The layout of our paper is as follows: In Sec. II we demonstrate how a generalized force law gives rise to a nonlinear diffusion coefficient in the continuum limit and derive such coefficients for a range of well-known force laws; in Sec. III we compare simulations of the continuum and discrete models; in Sec. IV we consider applications of the derived force law-diffusion coefficient relationship and examine the implications of a negative diffusion coefficient that arises from the Lennard-Jones model; and in Sec. V we conclude with a discussion and summary of our main results.

\section{MODEL DEVELOPMENT}

In this study we consider a population of homogeneous cells in one spatial dimension (see Fig. 1) in which the interaction between nearest neighbors is a function of the distance between their centers. In one spatial dimension a cell has two neighbors and the equation of motion of the $i$ th cell [Eq. (1)] can be written in the form

$$
\eta \dot{r}_{i}=F\left(r_{i}-r_{i-1}\right)-F\left(r_{i+1}-r_{i}\right), \quad i=1, \ldots, N .
$$

In order to obtain a continuum description of the cell dynamics described by Eq. (4), we introduce the variable $\Delta i$, where Eq. (4) can be thought of as the special case where $\Delta i=1$, and seek to obtain a governing equation of the form

$$
\hat{\eta} \dot{r}_{i}=\hat{F}\left(r_{i}-r_{i-\Delta i}\right)-\hat{F}\left(r_{i+\Delta i}-r_{i}\right), \quad i=1, \ldots, N,
$$

which describes the interaction of a volume element containing $\Delta i$ cells and centered at $r_{i}$ with neighboring elements centered at $r_{i-\Delta i}$ and $r_{i+\Delta i}$. Here, the careted variables $\hat{\eta}$ and $\hat{F}$ represent the damping constant of the volume element and the force between it and a neighboring element, respectively.

In order to progress we introduce the following physically motivated scaling relationships for $\hat{\eta}$ and $\hat{F}$ : the damping force on a volume element scales linearly with the number of cells in that volume such that $\hat{\eta}=\eta \Delta i$ (i.e., the damping

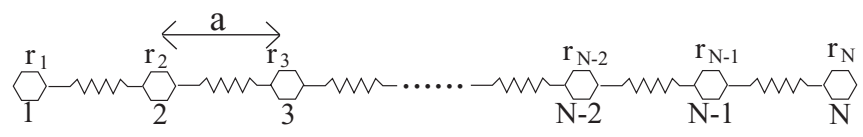

FIG. 1. A schematic illustration of a one-dimensional cell-based model. A one-dimensional chain of cells with cell positions $r_{i}(t)$ and cell labeling indices $i$ is depicted. In this schematic, the springs are in equilibrium and the distance between cells is the equilibrium length $a$. Note that cell labeling indices increase with distance from $r_{1}(t)$. 
constants of individual cells combine additively); and the force between two volume elements scales inversely with $\Delta i$ such that $\hat{F}(x \Delta i)=F(x) / \Delta i$ (this is a generalization of the calculation of the effective spring constant $k_{\text {eff }}$ of two linear springs connected in series with spring constants $k_{1}$ and $k_{2}$ such that $\left.1 / k_{\text {eff }}=1 / k_{1}+1 / k_{2}\right)$. Assuming that the spatial coordinates of the cell positions along the axis are a continuous function of $i$, i.e.,

$$
r_{i}(t)=r(i, t),
$$

the positions of nearest neighbors can be approximated using the Taylor expansions

$$
\begin{aligned}
& r_{i+\Delta i}=r_{i}+\frac{\partial r}{\partial i} \Delta i+\frac{1}{2} \frac{\partial^{2} r}{\partial i^{2}} \Delta i^{2}+O\left(\Delta i^{3}\right), \\
& r_{i-\Delta i}=r_{i}-\frac{\partial r}{\partial i} \Delta i+\frac{1}{2} \frac{\partial^{2} r}{\partial i^{2}} \Delta i^{2}+O\left(\Delta i^{3}\right),
\end{aligned}
$$

where the differentiability of $r$ is assumed. Substituting Eq. (7) into Eq. (5) and Taylor expanding $\hat{F}$ about $\partial r / \partial i \Delta i$, we obtain that

$$
\begin{aligned}
\hat{\eta} \frac{\partial r}{\partial t}= & \hat{F}\left(\frac{\partial r}{\partial i} \Delta i\right)+\hat{F}^{\prime}\left(\frac{\partial r}{\partial i} \Delta i\right)\left(-\frac{1}{2} \frac{\partial^{2} r}{\partial i^{2}} \Delta i^{2}+O\left(\Delta i^{3}\right)\right) \\
& +O\left(\Delta i^{4}\right)-\hat{F}\left(\frac{\partial r}{\partial i} \Delta i\right) \\
& -\hat{F}^{\prime}\left(\frac{\partial r}{\partial i} \Delta i\right)\left(\frac{1}{2} \frac{\partial^{2} r}{\partial i^{2}} \Delta i^{2}+O\left(\Delta i^{3}\right)\right)+O\left(\Delta i^{4}\right) .
\end{aligned}
$$

Using the postulated scaling relationships for $\hat{\eta}$ and $\hat{F}$, we obtain, upon cancellation and rearrangement, that

$$
\eta \frac{\partial r}{\partial t}=-F^{\prime}\left(\frac{\partial r}{\partial i}\right) \frac{\partial^{2} r}{\partial i^{2}}+O\left(\Delta i^{2}\right), \quad i=1, \ldots, N .
$$

Note that when $F$ is a linear function Eq. (9) takes a form similar to the Rouse model [16,28]. Furthermore, the error term is $O\left(\Delta i^{2}\right)$ owing to cancellation of the odd $O(\Delta i)$ terms and the assumed scalings of $\eta$ and $F$.

In order to reformulate Eq. (9) such that cell number density is the dependent variable, we make a coordinate transformation from the old independent variables $i$ and $t$ to the new independent variables $r$ (the dependent variable in the old coordinate system) and $\tau$ (time). The Jacobian of the coordinate transformation is

$$
\begin{aligned}
& \left(\begin{array}{ll}
\frac{\partial r}{\partial i} \mid t & \frac{\partial r}{\partial t} \mid i \\
\frac{\partial \tau}{\partial i} \mid t & \frac{\partial \tau}{\partial t} \mid i
\end{array}\right)=\left(\begin{array}{ll}
\frac{\partial i}{\partial r} \mid \tau & \frac{\partial i}{\partial \tau} \mid r \\
\frac{\partial t}{\partial r} \mid \tau & \frac{\partial t}{\partial \tau} \mid r
\end{array}\right)^{-1} \\
& =\frac{1}{\frac{\partial i}{\partial r \mid \tau} \frac{\partial t}{\partial \tau}\left|r-\frac{\partial i}{\partial \tau}\right| r \frac{\partial t}{\partial r} \mid \tau}\left(\begin{array}{cc}
\frac{\partial t}{\partial \tau} \mid r & -\frac{\partial i}{\partial \tau} \mid r \\
-\frac{\partial t}{\partial r} \mid \tau & \frac{\partial i}{\partial r} \mid \tau
\end{array}\right),
\end{aligned}
$$

and upon letting $t=\tau$ we can read off the relationships

$$
\frac{\partial r}{\partial i}_{\mid t}=\frac{1}{\frac{\partial i}{\partial r} \mid \tau}
$$

and

$$
\frac{\partial r}{\partial t}_{\mid i}=-\frac{\frac{\partial i}{\partial \tau} \mid r}{\frac{\partial i}{\partial r} \mid \tau}
$$

Substituting Eqs. (11) and (12) into Eq. (9) and rearranging yields

$$
\frac{\partial i}{\partial \tau}=\frac{-F^{\prime}\left(\frac{1}{\frac{\partial i}{\partial r}}\right)}{\eta\left(\frac{\partial i}{\partial r}\right)^{2}} \frac{\partial^{2} i}{\partial r^{2}},
$$

and after differentiating with respect to $r$ and defining the cell number density $q(r, \tau)=\frac{\partial i}{\partial r}$, we obtain that

$$
\frac{\partial q}{\partial \tau}=\frac{\partial}{\partial r}\left(D(q) \frac{\partial q}{\partial r}\right)
$$

with

$$
D(q)=-\frac{F^{\prime}\left(\frac{1}{q}\right)}{\eta q^{2}} .
$$

\begin{tabular}{|c|c|c|c|}
\hline Model & Force law $\left(F_{i j}\right)$ & Diffusion coefficient $[D(q)]$ & Reference \\
\hline General & $F_{L}^{i j}=F\left(\left|r_{i}-r_{j}\right|\right)$ & $D(q)=-\frac{F^{\prime}\left(\frac{1}{q}\right)}{\eta q^{2}}$ & \\
\hline Linear & $F_{L}^{i j}=k_{L}\left(a-\left|r_{i}-r_{j}\right|\right)$ & $D_{L}(q)=\frac{k_{L}}{\eta q^{2}}$ & {$[16,29,30]$} \\
\hline Cubic & $F_{C}^{i j}=k_{C}\left(a-\left|r_{i}-r_{j}\right|\right)^{3}$ & $D_{C}(q)=\frac{3 k_{C}}{\eta q^{2}}\left(a-\frac{1}{q}\right)^{2}$ & \\
\hline Linear-exponential & $F_{L E}^{i j}= \begin{cases}k\left(a-\left|r_{i}-r_{j}\right|\right), & \left|r_{i}-r_{j}\right|>d \\
k(a-d) \exp \left[k_{1}\left(d-\left|r_{i}-r_{j}\right|\right)\right], & \left|r_{i}-r_{j}\right|<d\end{cases}$ & $D_{L E}(q)= \begin{cases}\frac{k}{\eta q^{2}}, & q<\frac{1}{d} \\
\frac{k k_{1}(a-d)}{\eta q^{2}} \exp \left[k_{1}\left(d-\frac{1}{q}\right)\right], & q>\frac{1}{d}\end{cases}$ & {$[17]$} \\
\hline Hertz & $F_{H}^{i j}= \begin{cases}k_{H}\left(a-\left|r_{i}-r_{j}\right|\right)^{3 / 2}, & \left|r_{i}-r_{j}\right|<a \\
0, & \left|r_{i}-r_{j}\right|>a\end{cases}$ & $D_{H}(q)= \begin{cases}\frac{3 k_{H}}{2 \eta} \frac{(a-1 / q)^{\frac{1}{2}}}{q^{2}}, & q>\frac{1}{a} \\
0, & q \leqslant \frac{1}{a}\end{cases}$ & {$[11,14]$} \\
\hline Lennard-Jones & $F_{L J}^{i j}=k_{L J}\left(b \frac{\sigma^{m}}{\left|r_{i}-r_{j}\right|^{m+1}}-\frac{\sigma^{n}}{\left|r_{i}-r_{j}\right|^{n+1}}\right)$ & $D_{L J}(q)=\frac{k_{L J} \sigma^{n} q^{n}}{\eta}\left[b(m+1) \sigma^{m-n} q^{m-n}-(n+1)\right]$ & {$[18,19]$} \\
\hline
\end{tabular}

Thus the collective motion of cells in the one-dimensional chain can be described via a nonlinear diffusion equation in which the nonlinear diffusion coefficient scales linearly with the gradient of the force. We note that the validity of the continuum approximation depends on the accuracy of the Taylor expansions performed in Eqs. (7) and (8) with the underlying assumption being that there exists some intermediate scale $1 \ll \Delta i \ll N$ where the higher-order derivatives in $r$ can be neglected.

TABLE I. Force laws and corresponding nonlinear diffusion coefficients. In the Hertz model the parameter $k=\frac{4 E}{3 \sqrt{a}\left(1-v^{2}\right)}$, where $E$ represents the modulus of elasticity and $v$ the Poisson ratio. See Table II for parameter descriptions and scalings. 


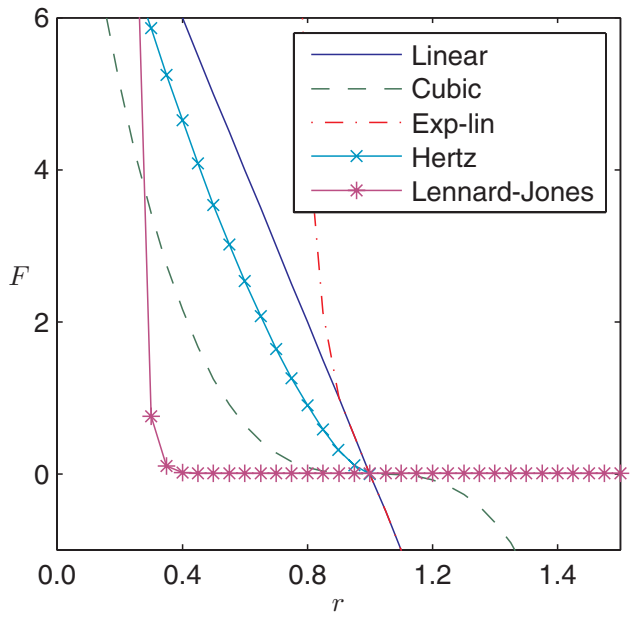

(a)

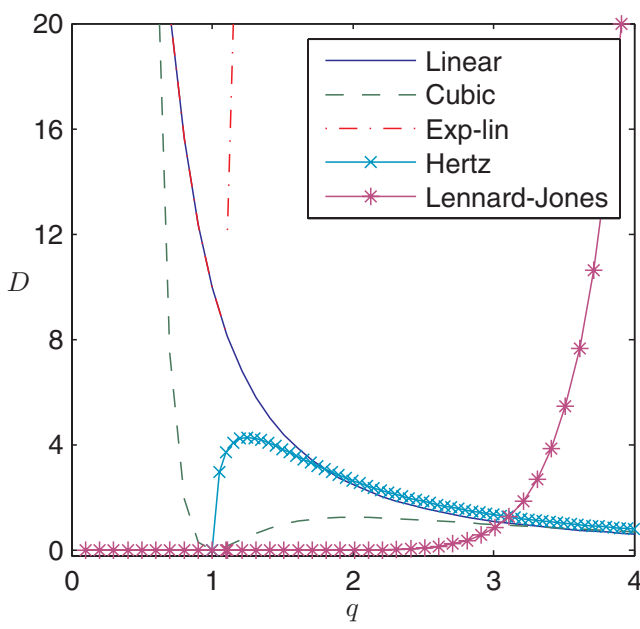

(b)

FIG. 2. (Color online) Comparison of force laws and corresponding diffusion coefficients. (a) The force between neighboring cells $F(r)$ plotted against their separation distance $r$. (b) The corresponding nonlinear diffusion coefficients $D(q)$ plotted against cell number density $q$. See Tables I and II for corresponding parameter values and units.

In Table I and Fig. 2 we present a range of particular force laws (linear, linear-exponential, Hertz, and Lennard-Jones) that have been used to simulate cell-cell interactions (the cubic force law has been included, as described below, in order to demonstrate that deviation from linearity in the discrete model yields markedly different behavior at the continuum scale). The parameters $a$ and $k$ represent the equilibrium separation distance between individual, neighboring cells and a mechanical rate constant, respectively, and we define the equilibrium separation and mechanical rate constant of a given volume element to be $\hat{a}$ and $\hat{k}$, respectively. We then assume the scaling relationship $\hat{a}=a \Delta i$ such that the equilibrium separation between two volume elements scales proportionally with the number of cells in a volume element. For the force laws presented in Table I, the scaling assumption made with regard to $F, \eta$, and $a$ are sufficient to determine the scaling of the $k$ 's (see Table II).

\section{MODEL ANALYSIS}

Before proceeding to a numerical comparison of the discrete force laws and their continuum counterparts, we make the following general observations. From a comparison of the diffusion coefficients corresponding to the linear and cubic

TABLE II. Parameter values used in the calculation of numerical solutions. Unit length is taken to be one cell diameter (c.d.) $\sim 10 \mu \mathrm{m}$, unit mass is taken to be the mass of a single cell (c.m.), and unit time is $1 \mathrm{~h}$. Parameter scalings with $\Delta i$ are indicated in parentheses.

\begin{tabular}{|c|c|c|c|}
\hline Parameter & Description & Value & Dimension \\
\hline$a(\Delta i)$ & Equilibrium length & 1 & c.d. \\
\hline$\eta(\Delta i)$ & Cell damping constant & 1 & $\mathrm{~h}^{-1} \mathrm{c} . \mathrm{m}$ \\
\hline$k_{L}(1 / \Delta i)$ & Linear spring constant & 15 & $\mathrm{~h}^{-2}$ c.m. \\
\hline$k_{C}\left(1 / \Delta i^{3}\right)$ & Cubic spring constant & 15 & $\mathrm{~h}^{-2}$ c.d. ${ }^{-2}$ c.m. \\
\hline$d(\Delta i)$ & Linear-exponential cutoff & 0.9 & c.d. \\
\hline$k_{1}(1 / \Delta i)$ & Rate of exponential force increase & 6 & c.d..$^{-1}$ \\
\hline$k_{H}\left(1 / \Delta i^{3 / 2}\right)$ & Hertz spring constant & 15 & $\mathrm{~h}^{-2}$ c.d. ${ }^{-1 / 2}$ c.m. \\
\hline$k_{L J}\left(1 / \Delta i^{n+1}\right)$ & Lennard-Jones spring constant & $1 e^{-8}$ & $\mathrm{~h}^{-2}$ c.d. ${ }^{2}$ c.m. \\
\hline$\sigma(\Delta i)$ & Lennard-Jones separation constant & $a b^{1 /(n-m)}$ & c.d. \\
\hline$m$ & Lennard-Jones parameter & 12 & Nondimensional \\
\hline$n$ & Lennard-Jones parameter & 6 & Nondimensional \\
\hline$b$ & Lennard-Jones parameter & 2 & Nondimensional \\
\hline$L$ & Domain length & 100 & c.d. \\
\hline$N$ & Number of cells & 150 & Nondimensional \\
\hline$N_{\text {nod }}$ & Number of nodes & 100 & Nondimensional \\
\hline$\rho$ & Steepness of initial data & 0.1 & c.d. ${ }^{-1}$ \\
\hline$r_{m}$ & Initial data parametrization & $L / 4$ & c.d. \\
\hline$r_{M}$ & Initial data parametrization & $3 L / 4$ & c.d. \\
\hline$\beta$ & Initial data parametrization & 1 & c.d. ${ }^{-1}$ \\
\hline
\end{tabular}




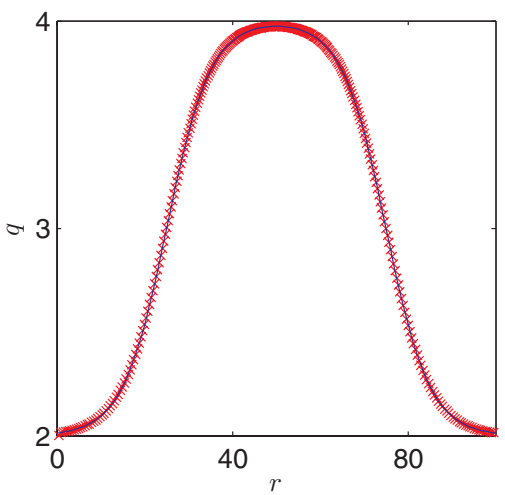

(a)

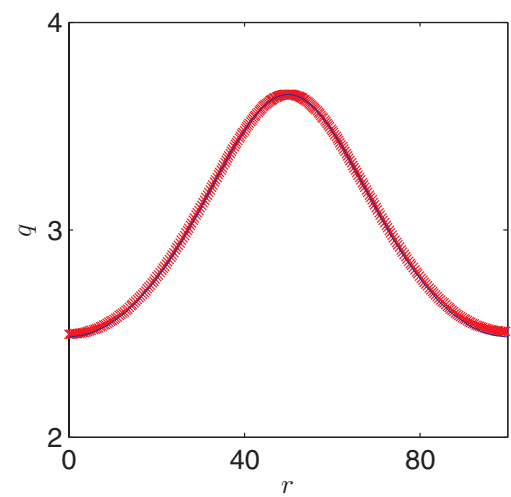

(d)

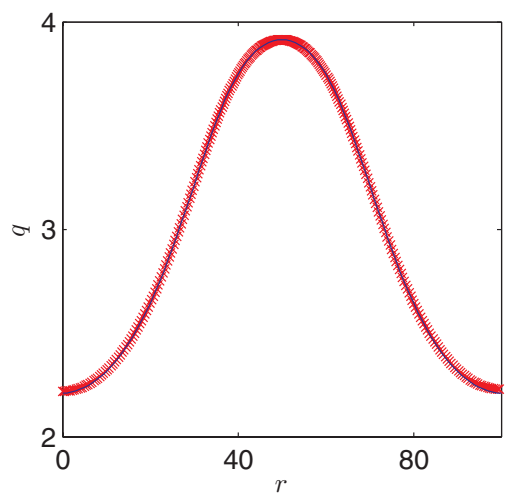

(b)

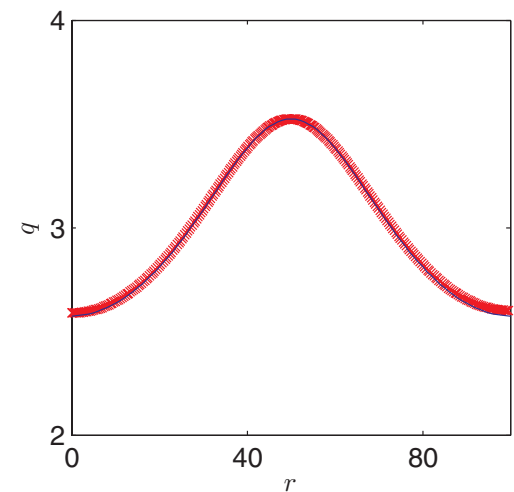

(e)

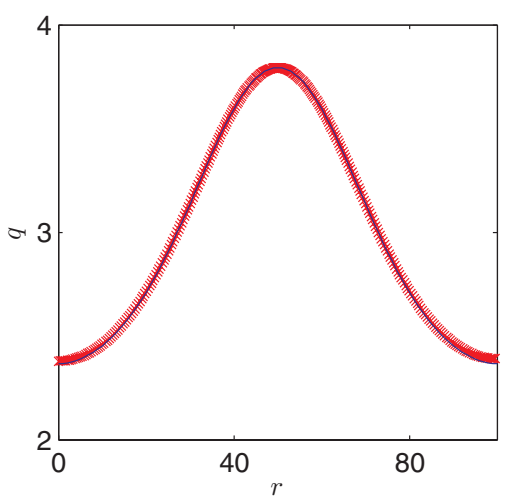

(c)

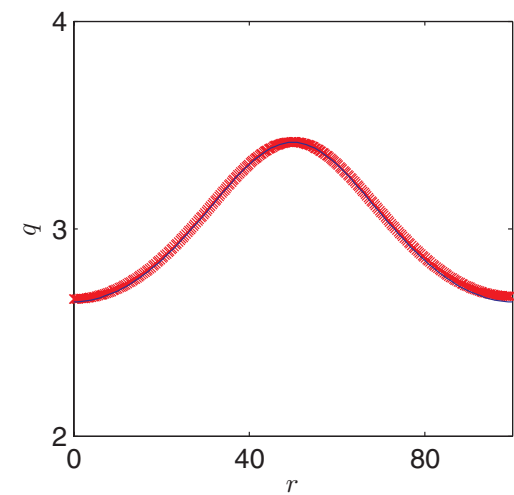

(f)

FIG. 3. (Color online) A comparison of discrete (marker) and continuum models with the linear force law at $t=\{0,48,96,144,192,240\}$. Cell number density $q(r, t)$ is plotted against $r$. Boundary and initial conditions given by Eqs. (16) and (17), respectively. $q_{\text {min }}=2$. Unit definitions and other parameter values as in Table II. See Table I for force law and diffusion coefficient.

force laws (see Table I), we expect markedly different behavior as the density tends to the equilibrium value $1 / a$. In the cubic model, as $q \rightarrow 1 / a, D \rightarrow 0$ and we expect to observe the formation of sharp fronts in the density profiles. Moreover, analysis of the derived diffusion coefficient for a given model can be instructive. For example, the Hertz model captures the elastic repulsion of neighboring cells. At low densities, cells do not exert forces upon one another, hence the diffusion coefficient is zero.

A further interesting feature of the general form of the diffusion coefficients is that they are not necessarily positive. In fact, for the Lennard-Jones model the diffusion coefficient is positive for large $q$ but negative for small $q$ (see Table I). Clearly, when the diffusion coefficient becomes negative the continuum description of the model becomes invalid as the continuum approximation made in Eq. (6) will not hold. We will return to this observation in the following section. We now compare numerical solutions of discrete and continuum models for different initial densities.

In order to validate the relationship between force laws and diffusion coefficients proposed in Eq. (15), we numerically simulated both discrete and continuum models for each force law listed in Table I using the parameter values listed in Table II. Here the discrete model is a one-dimensional chain of $N$ cells each moving with the velocity given by Eq. (1). The resulting set of ordinary differential equations (ODEs) was solved using the Runge-Kutta fourth- and fifth-order method solver ODE45 in MATLAB (The Mathworks Inc., Natwick, MA, USA). For the equivalent continuum model, Eq. (14) was solved for each of the corresponding nonlinear diffusion coefficients stated in Table I on the interval $r \in[0, L]$. No-flux boundary conditions

$$
\left.\frac{\partial q}{\partial r}\right|_{r=0}=\left.\frac{\partial q}{\partial r}\right|_{r=L}=0
$$

were applied at $r=0$ and $r=L$, respectively, and (arbitrarily) chosen initial conditions

$$
q(r, 0)=q_{\min }+\beta\left\{\tanh \left[\rho\left(r-r_{m}\right)\right]-\tanh \left[\rho\left(r-r_{M}\right)\right]\right\},
$$

where $q_{\min }, \rho, r_{m}, r_{M}$, and $\beta$ are parameters characterizing the initial distribution, were imposed. In order to calculate a numerical solution, the interval $[0, L]$ was discretized using a regular mesh with $N_{\text {nod }}$ nodes, where the spatial derivatives in Eq. (14) were approximated using finite differences, and we employed the method of lines to solve the resultant equations. The governing method-of-lines ODEs were solved in MATLAB using the ODE15s solver.

As an example of the temporal dynamics of solution behavior, in Fig. 3 we present simulation results from discrete and continuum models in the case of a linear force law 


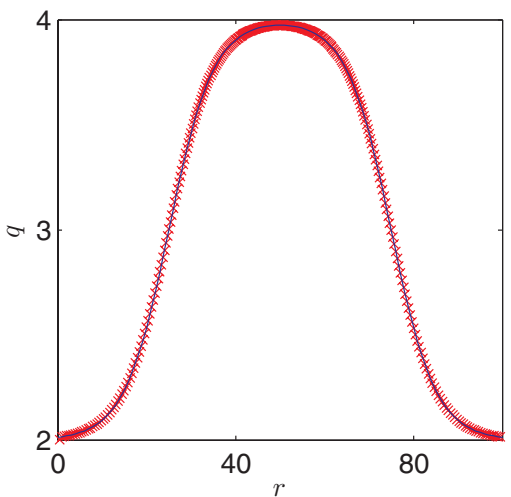

(a)

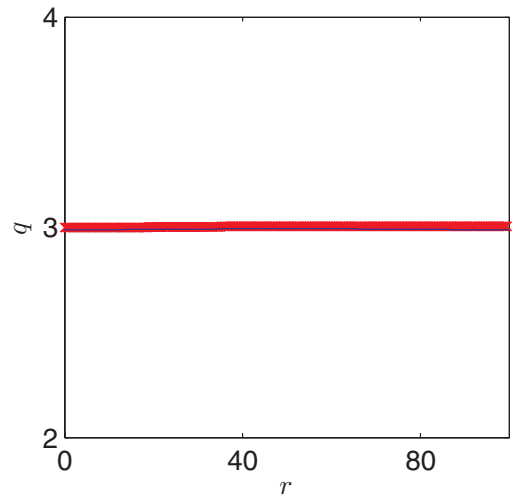

(d)

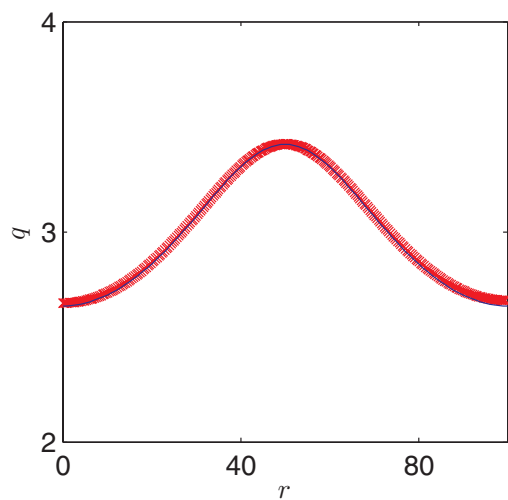

(b)

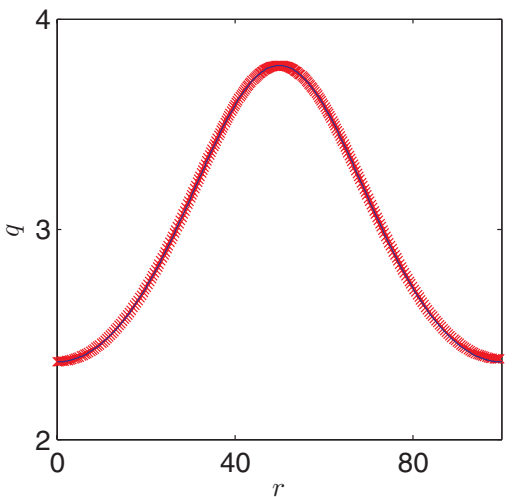

(e)

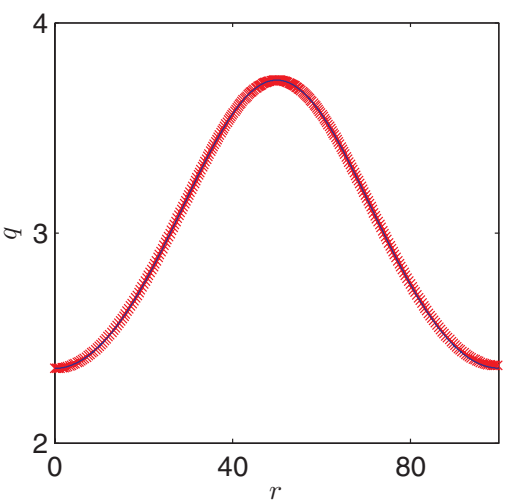

(c)

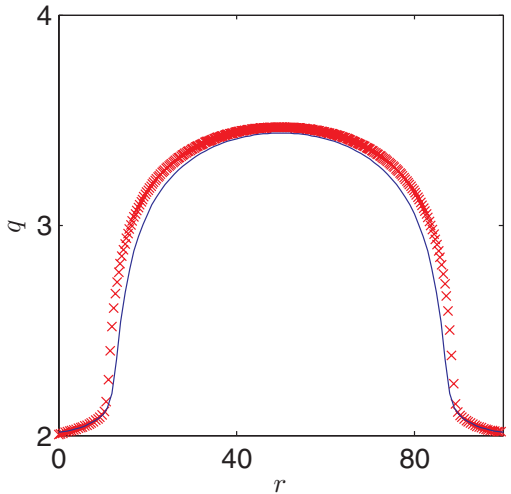

(f)

FIG. 4. (Color online) Simulations of discrete (markers) and continuum (solid lines) models with high initial densities $(N=600)$; all cells are initially compressed $(q>1)$. Cell number density $q(r, t)$ is plotted against $r$ at $t=100$. (a) Initial conditions; (b) linear force law, $k=30$; (c) cubic force law; (d) linear-exponential force law; (e) Hertz force law; (f) Lennard-Jones force law. Boundary and initial conditions given by Eqs. (16) and (17), respectively. $q_{\min }=2$. Unit definitions and other parameter values as in Table II. See Table I for respective force laws and diffusion coefficients.

as the initial data relax to an equilibrium configuration. Similar agreement is observed for the other force laws (results not shown). In order to highlight the significance of the nonlinearities in the diffusion coefficients presented in Table I, in Figs. 4-6 we compare discrete and continuum models when the maximum initial densities are high $\left(q_{\min }=2\right)$, intermediate $\left(q_{\min }=1\right)$, and low $\left(q_{\min }=1 / 2\right)$, respectively. In each of the figures cell densities are plotted against spatial coordinate $r$; the initial data are plotted in the top left figure, and the other figures are snapshots of the different models at (the arbitrarily chosen time point) $t=240$.

At high initial densities (see Fig. 4) the linear, cubic, and Hertz models exhibit qualitatively similar relaxation profiles while the linear-exponential model rapidly equilibrates as the initial high densities move the diffusion coefficient to the exponential regime. The Lennard-Jones model exhibits a mushroom-shaped profile as the diffusion coefficient is much larger in the center, where the density is high, than near the boundary. Discrete and continuum models are in excellent agreement.

At intermediate initial densities (see Fig. 5) we expect to observe qualitatively different behavior for each of the force laws. The linear model equilibrates at a faster rate than in the high-density case $\left(D \propto 1 / q^{2}\right)$ but the cubic model displays markedly different behavior (compare linear and cubic profiles) as a result of the density at the boundary tending to the equilibrium density and, hence, the diffusion coefficient tending to zero. The Hertz model behaves qualitatively similarly compared with the high-density case while the mushroom-shaped profile in the Lennard-Jones models is less pronounced, owing to the smaller initial densities.

At low initial densities (see Fig. 6) the linear model relaxes on a faster time scale than in the high- and intermediate-density cases. However, with the cubic force law $D \rightarrow 0$ as $q \rightarrow 1 / a$, and the assumption [made in Eq. (7)] of the existence of the derivative $\partial^{2} r / \partial i^{2}$ breaks down. Correspondingly, the derivative $\partial q / \partial r$ is not continuous as $q \rightarrow 1 / a$. In the calculation of the numerical solution in this case, conservation of cell number is enforced across an internal boundary defined at the spatial position where $q=1 / a$. Behavior in the Hertz model is qualitatively similarly to that of the cubic model at low densities, as again $D=0$ at interior points of the spatial domain. The simulations have been performed over a range of parameter space and similar results have been observed.

\section{APPLICATIONS}

We now examine how the derived relationship between force laws and their corresponding diffusion coefficients can 


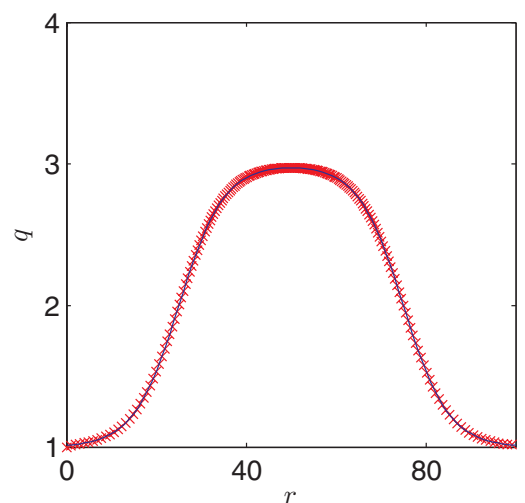

(a)

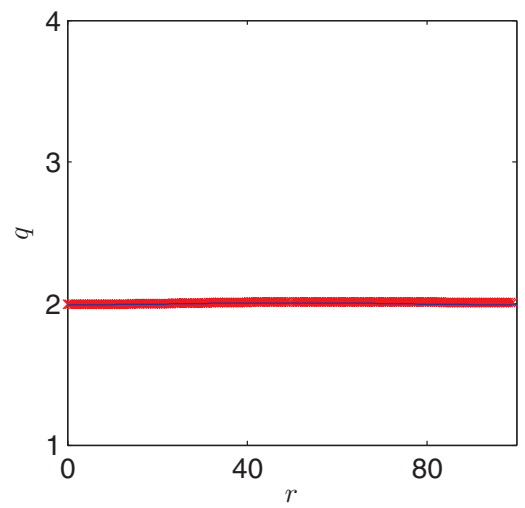

(d)

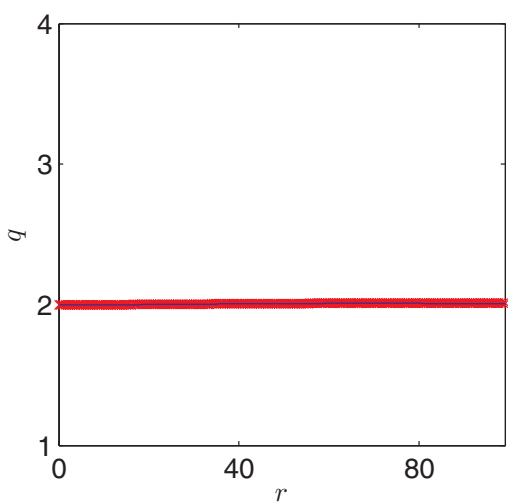

(b)

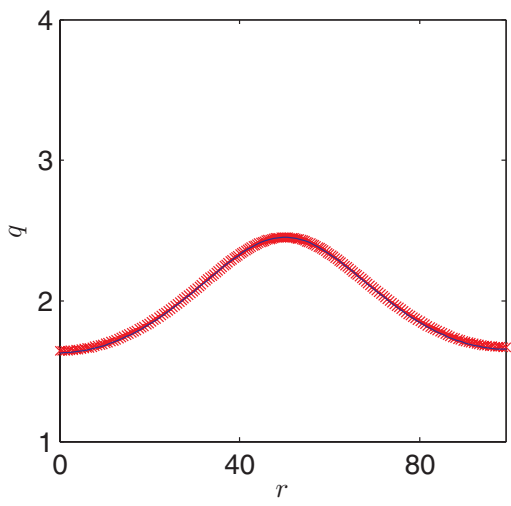

(e)

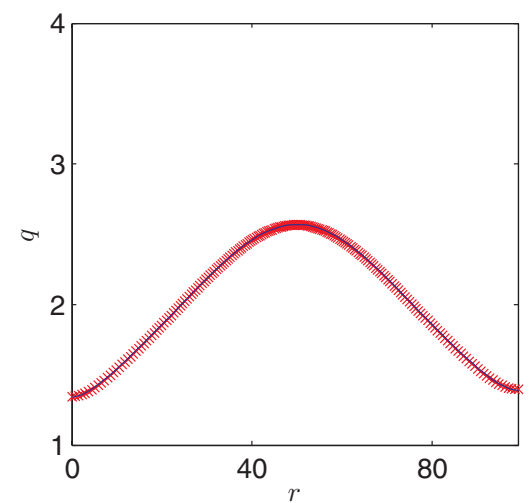

(c)

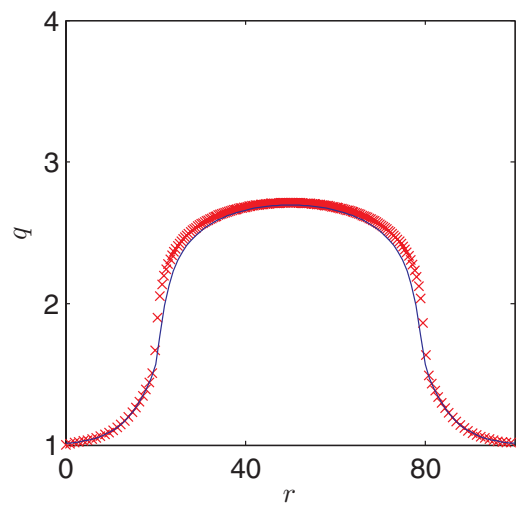

(f)

FIG. 5. (Color online) Simulations of discrete (markers) and continuum (solid lines) models with intermediate initial densities $(N=400)$; boundary cells are initially at equilibrium $(q=1)$ but interior cells are compressed $(q>1)$. Cell number density $q(r, t)$ is plotted against $r$ at $t=100$. (a) Initial conditions; (b) linear force law, $k=30$; (c) cubic force law; (d) linear-exponential force law; (e) Hertz force law; (f) Lennard-Jones force law, $k=1 e^{-7}$. Boundary and initial conditions given by Eqs. (16) and (17), respectively. $q_{\text {min }}=1$. Unit definitions and other parameter values as in Table II. See Table I for respective force laws and diffusion coefficients.

be used in a number of different contexts to relate models defined at different scales.

\section{A. Relating cellular Potts and off-lattice models}

Although Eq. (14) was derived by coarse-graining an underlying discrete model in order to determine a corresponding diffusion coefficient, this process can be inverted such that, given a diffusion coefficient, we can determine a corresponding force law at the cellular scale. In order to demonstrate the applicability of such an approach, we consider a coarse-grained description of the cellular Potts model in which Lushnikov et al. [22] have demonstrated that a nonlinear diffusion coefficient of the form

$$
D_{A}(q)=C \frac{1+\left(\frac{q}{q_{0}}\right)^{2}}{\left(1-\frac{q}{q_{0}}\right)^{2}},
$$

where $q_{0}=1 / a$, describes the evolution of cell densities in the underlying discrete simulations. Equating their diffusion coefficient Eq. (18) with the general form derived in Eq. (3) and integrating, we identify that a corresponding force law in the off-lattice framework is

$$
\begin{aligned}
F_{A}^{i j}(r)= & \eta C\left[\frac{2}{a} \ln \left(\frac{\left|r_{i}-r_{j}\right|}{\left|r_{i}-r_{j}\right|-a}\right)\right. \\
& \left.-\frac{3\left|r_{i}-r_{j}\right|-a}{\left(\left|r_{i}-r_{j}\right|-a\right)\left(\left|r_{i}-r_{j}\right|\right)}\right],
\end{aligned}
$$

where the integration constant has been set to zero such that the force between neighboring cells tends to zero as $\left|r_{i}-r_{j}\right|$ $\rightarrow \infty$. In Fig. 7(a) we plot the force law described by Eq. (19) against the separation distance between cells while the corresponding diffusion coefficient is plotted against cell number density in Fig. 7(b). The agreement between numerical solutions of the discrete and continuum models is similar to the cases presented in Figs. 4-6 (results not shown).

The key benefit of the force law derived in Eq. (19) is that, via the consideration of continuum limits, we have coarse-grained from a higher spatial resolution of a cell in the cellular Potts model (each cell is represented by multiple lattice sites) to a lower resolution in the off-lattice, cell-based model (each cell is represented by a single point) while still retaining the correct cellular Potts behavior in the continuum limit. The force law describes how individual cells interact with each other and important features of individual cell behavior in the cellular Potts framework can be extracted from it. For example, 


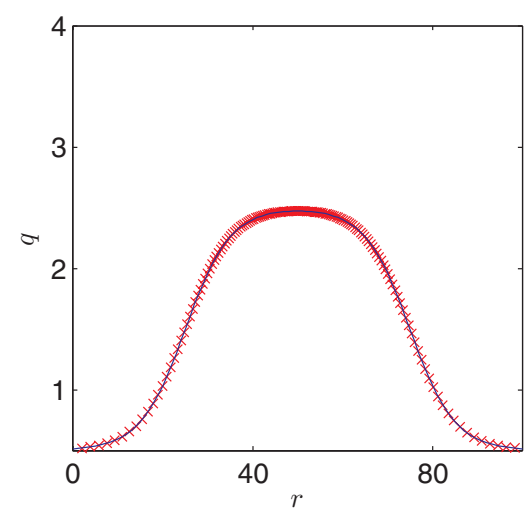

(a)

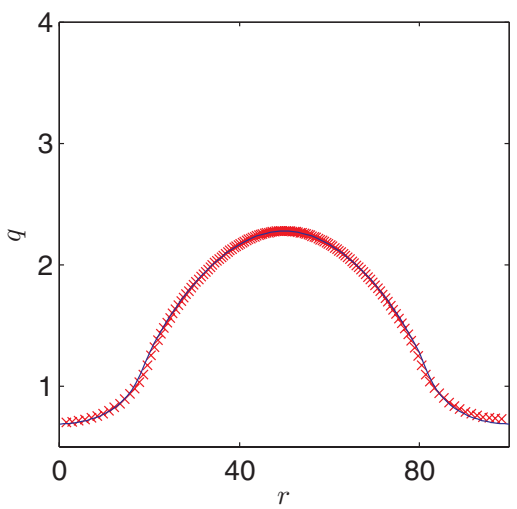

(d)

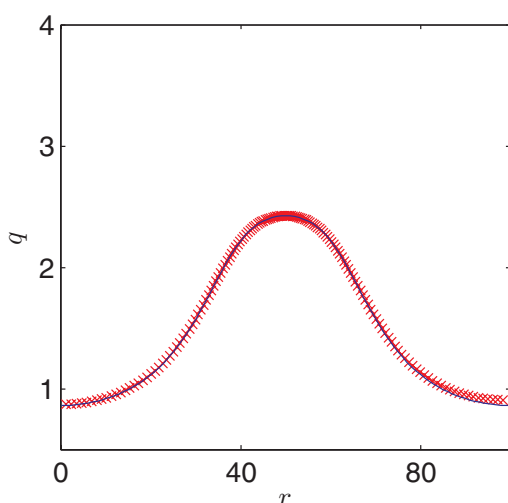

(b)

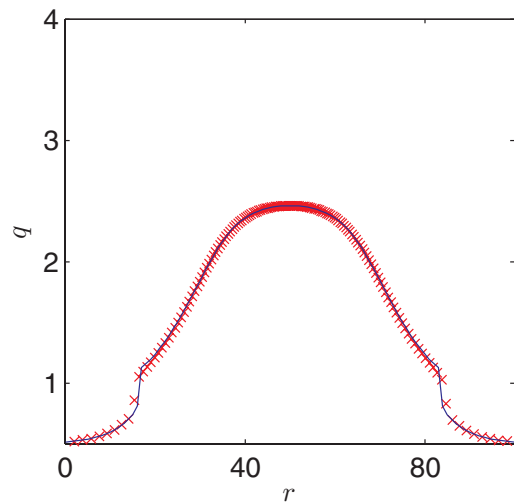

(e)

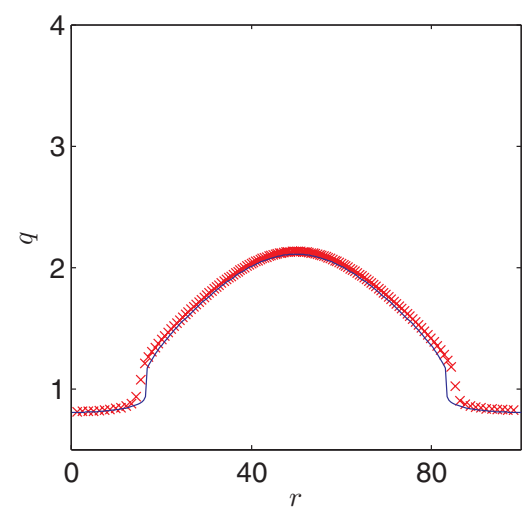

(c)

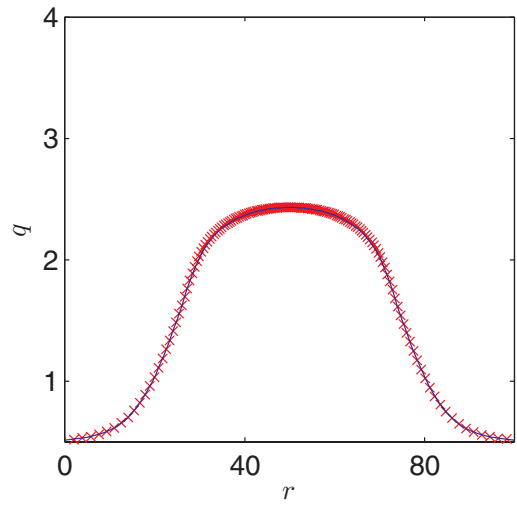

(f)

FIG. 6. (Color online) Simulations of discrete (markers) and continuum (solid lines) models with low initial densities $(N=300)$; boundary cells are initially stretched beyond equilibrium $(q<1)$ while interior cells are compressed $(q>1)$. Cell number density $q(r, t)$ is plotted against $r$ at $t=100$. (a) Initial conditions; (b) linear force law, $k=30$; (c) cubic force law; (d) linear-exponential force law; (e) Hertz force law; (f) Lennard-Jones force law, $k=1 e^{-6}$. Boundary and initial conditions given by Eqs. (16) and (17), respectively. $q_{\min }=1 / 2$. Unit definitions and other parameter values as in Table II. See Table I for respective force laws and diffusion coefficients.

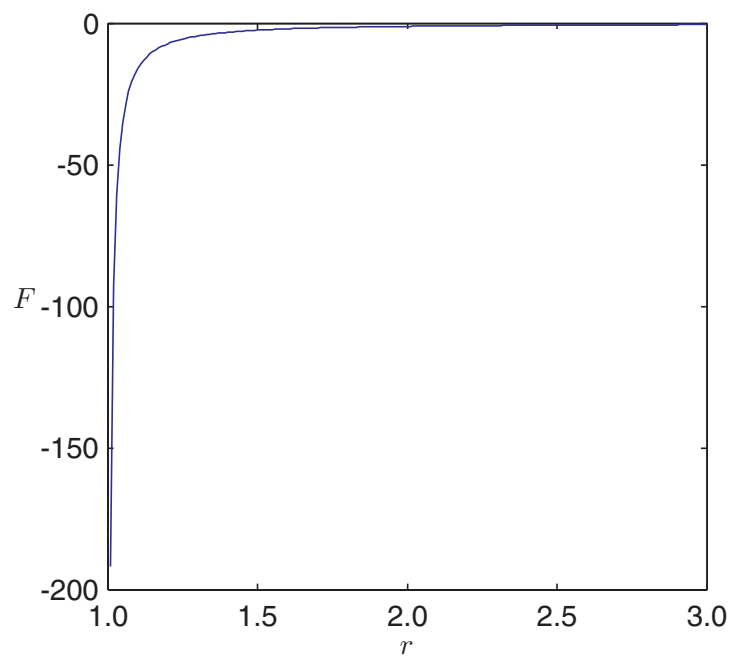

(a)

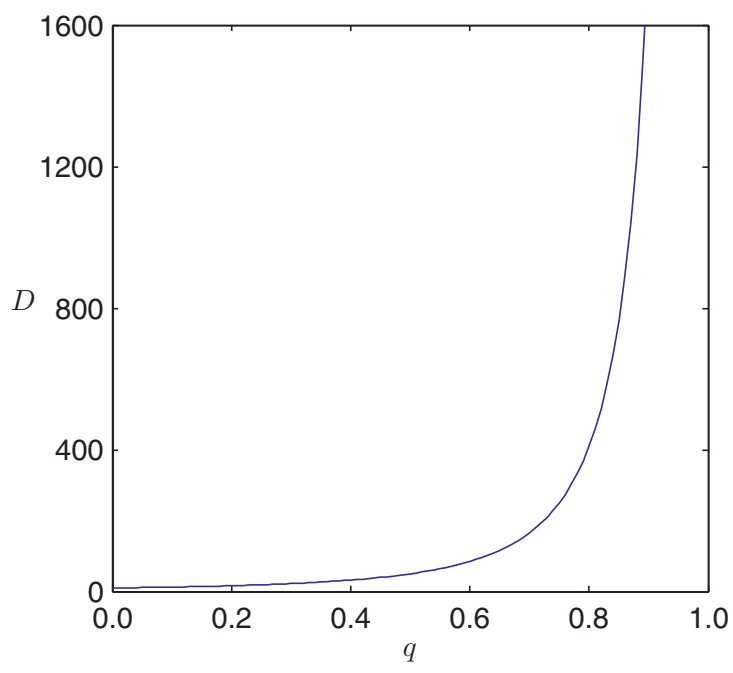

(b)

FIG. 7. (Color online) The nonlinear force law and diffusion coefficients corresponding to the cellular Potts model. (a) The force $F(r)$ [Eq. (19)] is plotted against cell separation $r$. (b) The nonlinear diffusion coefficient $D(q)$ [Eq. (18)] previously derived by Lushnikov $e t$ al. [22] plotted against cell number density $q . C=1$. Unit definitions and other parameter values as in Table II. 


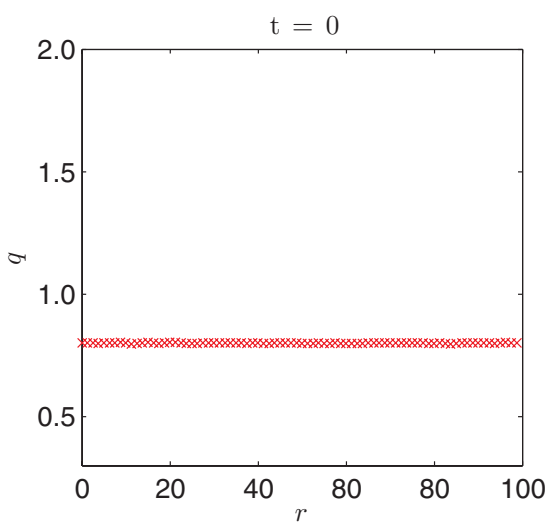

(a)

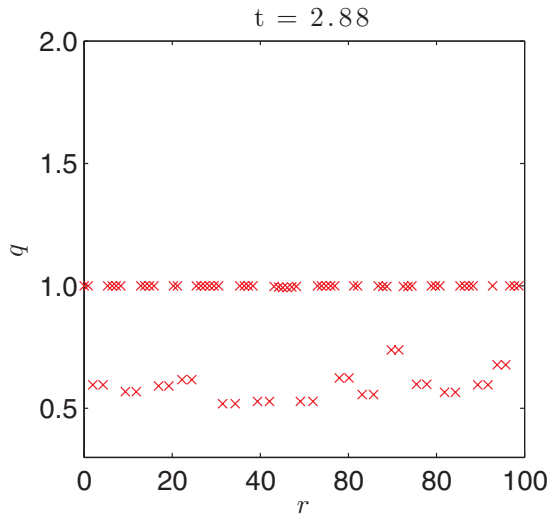

(d)

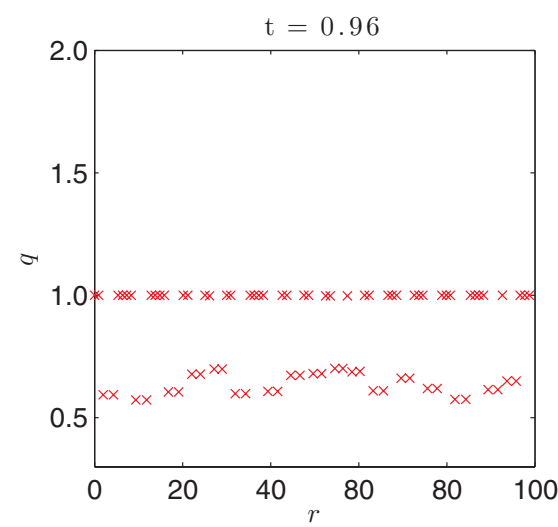

(b)

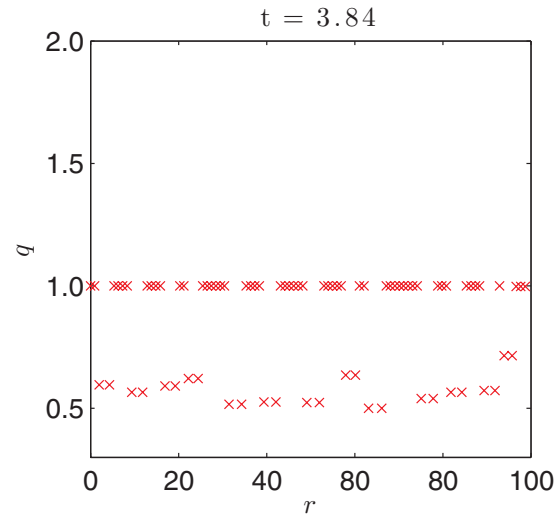

(e)

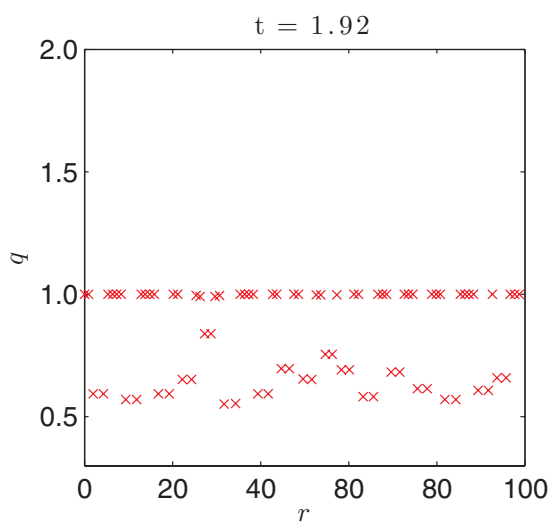

(c)

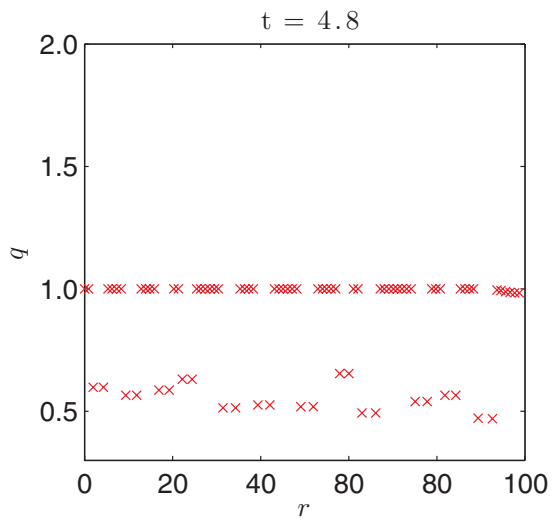

(f)

FIG. 8. (Color online) Aggregation in the Lennard-Jones model. Simulations of discrete model (markers) with uniform initial density $(N=80, L=100)$. Cell number density $q(r, t)$ is plotted against $r$ with increasing $t$. $k=0.1$. Boundary conditions given by Eq. (16). Unit definitions and other parameter values as in Table II. See Table I for force law and diffusion coefficient.

as a result of volume exclusion cells cannot come within a distance of $a$ from each other. In fact, as their separation distance approaches $a$, the repulsive force between them tends to infinity, whereas when the cells are widely separated they can move independently of one another and the diffusion coefficient is then constant.

\section{B. Relating nonlinear diffusion coefficients to force laws}

Equation (3) can be used to relate diffusion coefficients defined in a phenomenological manner at the population scale to discrete force laws. For example, in order to model population-scale random movement in ecology, Murray [31] considered diffusion equations with nonlinear coefficients of the form

$$
D(q)=k q^{m},
$$

with $m>0$. Using Eq. (3) we can determine a class of individual-level models that can give rise to such populationscale behaviors and find that the corresponding force law takes the form

$$
F_{i j}=-\frac{k}{(m+1)\left|r_{i}-r_{j}\right|^{m+1}} .
$$

Thus a constant diffusion coefficient arises from considering cells which interact via an inverse force law $(m=0)$ while a diffusion coefficient that varies linearly with density (often described as a porous medium equation) arises from an inverse square repulsion. The agreement between numerical solutions of the discrete and continuum models is similar to the cases presented in Figs. 4-6 (results not shown). We note that the constant diffusion coefficient arises from an inverse force law; hence a constant diffusion need not, as is often assumed, represent random collisions between interacting bodies but rather can also arise from a local repulsion between nearest neighbors that decays inversely with distance.

\section{Aggregation in the Lennard-Jones model}

In Sec. III we examined the behavior of the LennardJones model when $k \ll 1$ such that the negative component of the diffusion coefficient at low densities was negligibly small. We now relax this assumption and investigate model behavior when $k=O(1)$. In this regime the negative diffusion coefficient plays a dominant role in cell dynamics and we expect the validity of the continuum model to break down. In Fig. 8 we present simulation results from the discrete model which demonstrate aggregation. As cell densities are initially less than 1 , the diffusion coefficient is negative and 
cells move up gradients in their density. Thus an initially continuous cell distribution becomes discontinuous as the cell population splits into aggregates. Cells continue to move up density gradients until the density reaches 1 , at which point the diffusion coefficient becomes positive and the continuum model becomes valid for each of the separate aggregates. Hence, force laws of Lennard-Jones type can provide a mechanism for cell aggregation that is dependent on local interactions alone. We note that a qualitatively similar diffusion coefficient to the one derived in this study has been proposed by Mertens et al. [32] in a model that describes the one-dimensional directed self-assembly of nanoparticles.

\section{SUMMARY AND DISCUSSION}

In a previous work [16], we described how a onedimensional chain of overdamped cells interacting via a linear force law can be described by a nonlinear diffusion equation for cell number density in the continuum limit. The nonlinear diffusion coefficient allowed behavior in the underlying simulations to be categorized as well as a qualitative comparison of that particular model with the cellular Potts model via comparison of their respective diffusion coefficients.

In this study we have extended our approach to describe how a nonlinear diffusion coefficient can be derived for a more general force law. The result from the previous study then falls out as a particular case of the new result (a linear force law), but we also derive specific diffusion coefficients for a range of commonly used nonlinear force laws. We demonstrate how a comparison of the functional forms of the different diffusion coefficients can lend insight into the behavior of simulations with the different force laws.

In order to demonstrate the accuracy of the continuum approach, we compared numerical results from discrete and corresponding continuum models and observed excellent agreement. The relaxation profiles of the cell populations depended strongly on the magnitude of the initial cell density and the type of force law being considered. For example, the cubic force law model behaved qualitatively similarly to the linear model at high initial densities but at low densities showed markedly different qualitative behavior. Both qualitative and quantitative features of the different simulations were captured using the continuum model.

We have claimed that one of the benefits of deriving coarsegrained models is that it allows one to systematically classify discrete simulations. In previous work we used the coarsegrained nonlinear diffusion coefficients to make a qualitative comparison between the off-lattice, linear spring model and the cellular Potts model. Using the general form of the nonlinear diffusion coefficient derived in this paper, we have determined a nonlinear force law which gives the same coarse-grained behavior. This procedure allows us to transform from a cellular
Potts description of a cell population, where a single cell is represented by many lattice sites, to an off-lattice, cell-based description, where a single cell is represented by a single point.

Using a similar approach we can define force laws that yield particular classes of phenomenologically derived diffusion coefficients. As an example, we consider a particular form of diffusion coefficient proposed by Murray [31] that is used to describe the movement of populations in ecology. Using the derived force law-diffusion coefficient relationship in this study, we then demonstrate that Murray's diffusion coefficients have a direct correspondence with inverse force laws. This result provides a justification for the use of linear diffusion even if individuals are not performing unbiased random walks.

Diffusion coefficients derived using a bottom-up approach can lead to interesting, and perhaps unexpected, strongly nonlinear behavior at the population scale. This is exemplified by the diffusion coefficient corresponding to the LennardJones force law which becomes negative at low densities, resulting in the breakdown of the continuum model when $k$ is sufficiently large. In this limit, cells move up density gradients, and an initially homogeneous low-density distribution of cells forms a series of microaggregates of higher densities; in each of the separate microaggregates density distributions are continuous. We note that Bodnar and Velazquez [25] have described similar model behaviors for the case of general attractive potentials.

Fozard et al. [24] have considered the continuum limit of a cell vertex model in one spatial dimension. We note that, upon omission of the cell-cell friction included in the discrete vertex model, their continuum equation governing cell number density can be posed as a nonlinear diffusion problem similar to that described by Murray et al. [16]. We expect that their analysis extends to the case of the nonlinear force laws presented in this study via an appropriate choice for the relationship between pressure and cell density.

The hypothesis that diffusion coefficients can be used to characterize and classify different discrete, cell-based simulations has gained traction in recent years, primarily owing to the derivation of different types of diffusion coefficients across a range of disparate systems [16,21,22,25-27]. In this study we add further weight to this hypothesis by describing how off-lattice cell-based models can give rise to a particular class of nonlinear diffusion coefficient. It remains to be discovered how these results generalize to higher spatial dimensions.

\section{ACKNOWLEDGMENTS}

P.J.M. acknowledges the support of the Engineering and Physical Sciences Research Council and St Hugh's College, Oxford. P.K.M. was partially supported by a Royal SocietyWolfson Research Merit Award. M.J.T. was supported by a Research Council UK fellowship.
[1] J. von Neumann, in The Theory of Self-reproducing Automata, edited by A. Burks (University of Illinois Press, Champaign, 1966).

[2] T. Alarcón, H. M. Byrne, and P. K. Maini, J. Theor. Biol. 225, 257 (2003).
[3] A. R. A. Anderson, Math. Med. Biol.22, 163 (2005).

[4] A. Deutsch and S. Dormann, Cellular Automaton Modelling of Biological Pattern Formation (Birkhauser, Basel, 2005).

[5] W. Düchting and G. Dehl, J. Biomed. Eng. 2, 167 (1980). 
[6] K. Smallbone, R. A. Gatenby, R. J. Gillies, D. J. Gavaghan, and P. K. Maini, J. Theor. Biol. 244, 703 (2007).

[7] M. V. Plikus, J. A. Mayer, D. de La Cruz, R. E. Baker, P. K. Maini, R. Maxson, and C. M. Chuong, Nature (London) 451, 340 (2008).

[8] F. Graner and J. A. Glazier, Phys. Rev. Lett. 69, 2013 (1992).

[9] D. Drasdo and S. Hohme, Phys. Biol.2, 133 (2005).

[10] D. Drasdo and M. Loeffler, Nonlinear Anal. Theory, Methods Appl. 47, 245 (2001).

[11] J. Galle, M. Loeffler, and D. Drasdo, Biophys. J. 88, 62 (2005).

[12] F. Meineke, C. S. Potten, and M. Loeffler, Cell Proliferation 34, 253 (2001).

[13] G. Schaller and M. Meyer-Hermann, J. Theor. Biol. 247, 554 (2007).

[14] G. Schaller and M. Meyer-Hermann, Phys. Rev. E 71, 051910 (2005).

[15] I. M. M. van Leeuwen, G. R. Mirams, A. Walter, A. Fletcher, P. Murray, J. Osborne, S. Varma, S. J. Young, J. Cooper, B. Doyle, J. M. Pitt-Francis, L. Momtahan, P. Pathmanathan, J. P. Whiteley, S. J. Chapman, D. J. Gavaghan, O. E. Jensen, J. R. King, P. K. Maini, S. L. Waters, and H. M. Byrne, Cell Proliferation 42, 617 (2009).

[16] P. J. Murray, C. M. Edwards, M. J. Tindall, and P. K. Maini, Phys. Rev. E 80, 031912 (2009).

[17] P. Pathmanathan, J. Cooper, A. Fletcher, G. Mirams, P. Murray, J. Osborne, J. Pitt-Francis, A. Walter, and S. J. Chapman, Phys. Biol. 6, 036001 (2009).
[18] J. Jeon, V. Quaranta, and P. T. Cummings, Biophys. J. 98, 37 (2010).

[19] C. R. Sweet, S. Chatterjee, Z. Xu, K. Bisordi, E. D. Rosen, and M. Alber, J. R. Soc., Interface 8, 1760 (2011).

[20] R. Erban and H. G. Othmer, SIAM J. Appl. Math. 65, 361 (2004).

[21] M. Alber, N. Chen, P. M. Lushnikov, and S. A. Newman, Phys. Rev. Lett. 99, 168102 (2007).

[22] P. M. Lushnikov, N. Chen, and M. Alber, Phys. Rev. E 78, 061904 (2008).

[23] H. M. Byrne and D. Drasdo, J. Math. Biol. 58, 657 (2009).

[24] J. A. Fozard, H. M. Byrne, O. E. Jensen, and J. R. King, Math. Med. Biol. 27, 39 (2009).

[25] M. Bodnar and J. J. L. Velazquez, Math. Methods Appl. Sci. 28, 1757 (2005)

[26] M. J. Simpson, K. A. Landman, B. D. Hughes, and A. E. Fernando, Physica A 389, 1412 (2010).

[27] A. E. Fernando, K. A. Landman, and M. J. Simpson, Phys. Rev. E 81, 011903 (2010).

[28] P. E. Rouse, J. Chem. Phys. 21, 1272 (1953).

[29] P. J. Murray, A. Walter, A. G. Fletcher, C. M. Edwards, M. J. Tindall, and P. K. Maini, Phys. Biol. 8, 026011 (2011).

[30] P. J. Murray, J. W. Kang, G. R. Mirams, S. Y. Shin, H. M. Byrne, P. K. Maini, and K. H. Cho, Biophys. J. 99, 716 (2010).

[31] J. D. Murray, in Mathematical Biology, edited by S. S. Antman, J. E. Marsden, L. Sirovich, and S. Wiggins (Springer, Berlin, 1989).

[32] K. Mertens, V. Putkaradze, D. Xia, and S. R. J. Brueck, J. Appl. Phys. 98, 034309 (2005). 